\title{
Observation of an Aligned Gas - Solid "Eutectic" during Controlled Directional Solidification aboard the International Space Station - Comparison with Ground-based Studies
}

\author{
R.N. Grugel ${ }^{1}$ and A.V. Anilkumar ${ }^{2}$ \\ ${ }^{1}$ Marshall Space Flight Center, Huntsville, AL 35812 \\ ${ }^{2}$ Vanderbilt Univ., Dept. of Mech. Engineering, Nashville, TN - 37235
}

Direct observation of the controlled melting and solidification of succinonitrile was conducted in the glovebox facility of the International Space Station (ISS). The experimental samples were prepared on ground by filling glass tubes, $1 \mathrm{~cm} I D$ and approximately $30 \mathrm{~cm}$ in length, with pure succinonitrile (SCN) in an atmosphere of nitrogen at 450 millibar pressure for eventual processing in the Pore Formation and Mobility Investigation (PFMI) apparatus in the glovebox facility (GBX) on board the ISS.

Real time visualization during controlled directional melt back of the sample showed nitrogen bubbles emerging from the interface and moving through the liquid up the imposed temperature gradient. Over a period of time these bubbles disappear by dissolving into the melt. Translation is stopped after melting back of about $9 \mathrm{~cm}$ of the sample, with an equilibrium solidliquid interface established.

During controlled re-solidification, aligned tubes of gas were seen growing perpendicular to the planar solid/liquid interface, inferring that the nitrogen previously dissolved into the liquid SCN was now coming out at the solid/liquid interface and forming the little studied liquid = solid + gas eutectic-type reaction. The observed structure is evaluated in terms of spacing dimensions, interface undercooling, and mechanisms for spacing adjustments. Finally, the significance of processing in a microgravity environment is ascertained in view of ground-based results.

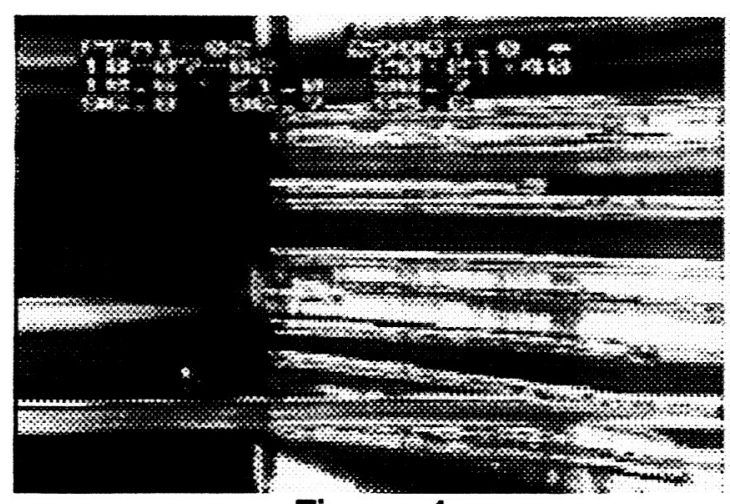

Figure 1

Figure 1: Observation of a planar interface showing the liquid (left) to solid + gas (right) transition. $\mathrm{V}=4 \mathrm{microns} /$ second, $\mathrm{G}=25.5 \mathrm{~K} / \mathrm{cm}$.

Richard N. Grugel, Ph.D.

Marshall Space Flight Center

MS - SD46

Huntsville, AL 35812 